\title{
JAK3 and TYK2 Serve as Prognostic Biomarkers and Are Associated with Immune Infiltration in Stomach Adenocarcinoma
}

\author{
Lingkai Meng, ${ }^{1}$ Ling Ding, ${ }^{2}$ Yue $\mathrm{Yu}^{3}$ and Wang Li ${ }^{1}{ }^{1}$ \\ ${ }^{1}$ NHC Key Laboratory of Hormones and Development, Tianjin Key Laboratory of Metabolic Diseases, Chu Hsien-I Memorial \\ Hospital \& Tianjin Institute of Endocrinology, Tianjin Medical University, Tianjin 300134, China \\ ${ }^{2}$ Department of Anesthesiology, Tianjin Union Medical Center, Tianjin 300191, China \\ ${ }^{3}$ University of Alberta, Edmonton, Alberta, Canada
}

Correspondence should be addressed to Wang Li; lwbaker@126.com

Received 1 July 2020; Revised 19 August 2020; Accepted 3 September 2020; Published 6 October 2020

Academic Editor: Tao Huang

Copyright (c) 2020 Lingkai Meng et al. This is an open access article distributed under the Creative Commons Attribution License, which permits unrestricted use, distribution, and reproduction in any medium, provided the original work is properly cited.

Background. Stomach adenocarcinoma (STAD) is one of the most common malignant tumors. The Janus kinases (JAKs) play a significant part in cellular biological process, inflammation, and immunity. The roles of JAKs in STAD are still not systematically described. Methods. A series of bioinformatics tools were used to clarify the role of JAKs in STAD. Results. JAK3/TYK2 levels were significantly increased in STAD during subgroup analyses based on gender, tumor grade, cancer stages, and nodal metastasis status. STAD patients with high levels of JAK3/TYK2 had poor overall survival, postprogression survival, and first progression. Immune infiltration revealed a significant correlation between JAK3/TYK2 expression and the abundance of immune cells as well as immune biomarker expression in STAD. JAK3/TYK2 was associated with the adaptive immune response, chemokine signaling pathway, and JAK-STAT signaling pathway. Conclusions. JAK3 and TYK2 serve as prognostic biomarkers and are associated with immune infiltration in STAD.

\section{Introduction}

Gastric cancer (GC) is one of the most common malignant tumors, with the fifth largest incidence and third largest mortality rate among all malignant tumors [1]. Stomach adenocarcinoma (STAD) is the most common subtype of GC, accounting for over $95 \%$ of all GC cases.

Although the identification of Helicobacter pylori has reduced the incidence of gastric cancer, it is estimated that $1,033,701$ patients would be initially diagnosed with GC worldwide in 2018 [2]. Moreover, the molecular mechanisms concerning the tumorigenesis and progress of GC is far from clarified and the therapeutic measures for GC are limited, resulting in a poor patient prognosis. Furthermore, the overall survival of patients with advanced or metastatic GC is only approximately 1 year [3]. These sobering data illustrate a critical need for novel prognostic biomarkers and therapeutic targets for STAD.
Janus kinases (JAKs) are major activators of signal transducers and play a significant role in cellular biological processes, inflammation, and immunity [4-7]. JAK/STAT signaling is a key regulator of gene expression, transcriptional programs, and immune response. In all, four members have been identified in the JAK family: JAK1/2/3 and TYK2. Genetic alterations of JAKs are involved in tumor cell proliferation, migration, apoptosis, and metastasis in certain types of cancers [8]. Increasing evidence demonstrates JAKs as a prognostic biomarker and therapeutic target for many cancers or other diseases, such as JAK3 for renal cell carcinoma, JAK2 for acute lymphoblastic leukemia [9], JAK2 for skin cutaneous melanoma [10], and TYK2 for hepatocellular carcinoma [11]. However, specific functions of JAKs in STAD remain to be systematically described.

Therefore, we aimed to explore the expression of JAKs and prognostic value of the association between immune infiltration and JAKs in STAD. We further evaluated the cor- 

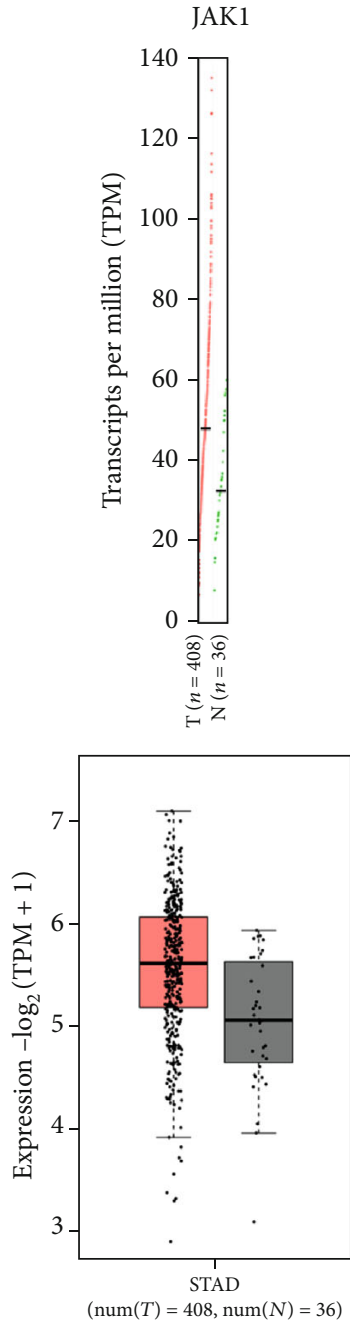

(a)
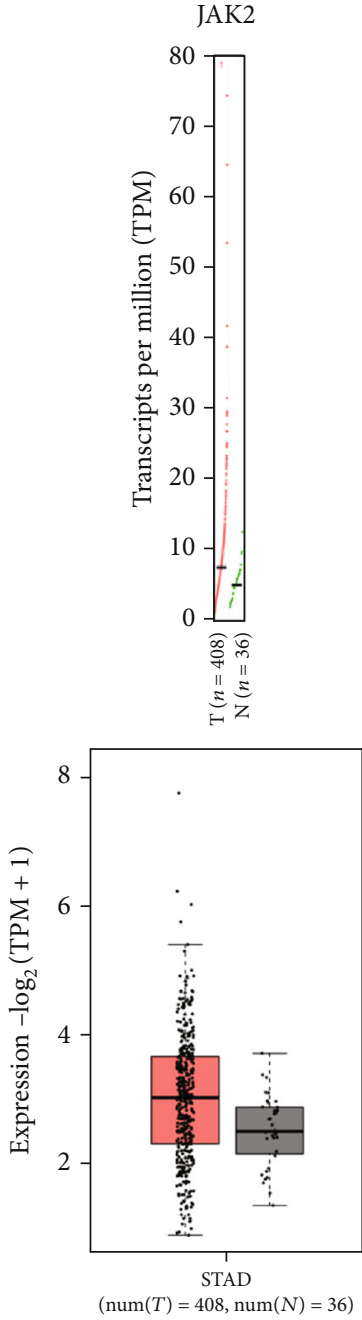

(b)
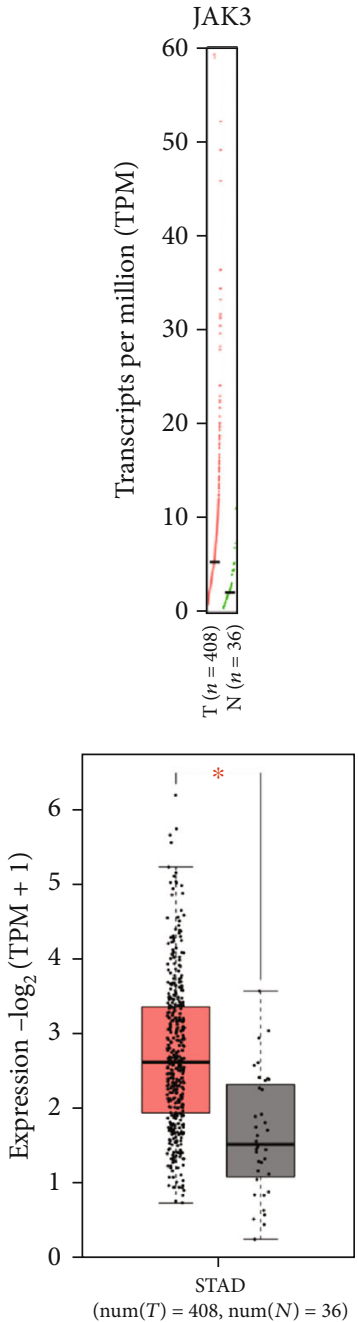

(c)
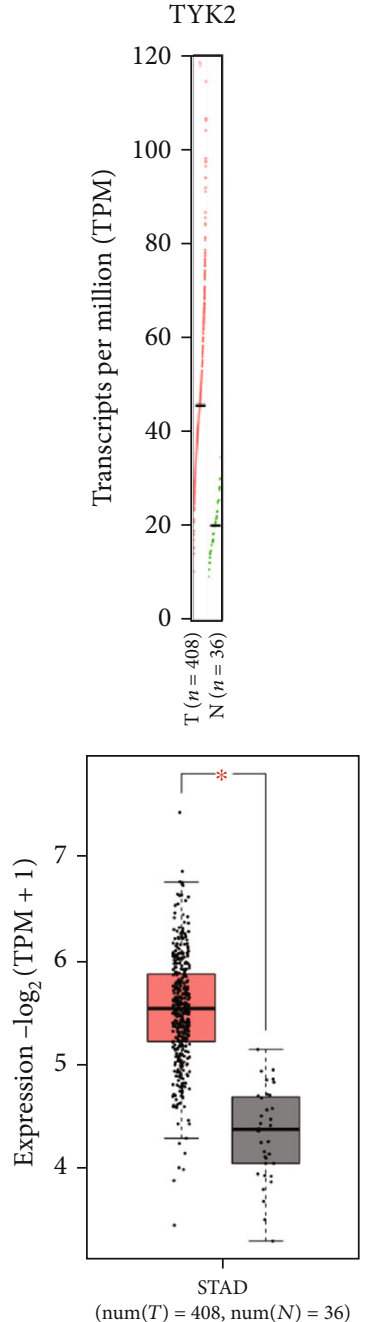

(d)

FIGURE 1: The expression of JAKs in STAD (GEPIA). The expression of JAK3 and TYK2 were significantly elevated in STAD tissues at mRNA level. STAD: stomach adenocarcinoma; ${ }^{*} P<0.05$; $\mathrm{T}$ : tumor tissues; $\mathrm{N}$ : normal tissues.

relation between JAK expression and the clinicopathological parameters of patients as well as immune infiltration in STAD. Our results may provide additional evidence about the prognostic biomarkers and therapeutic targets for STAD.

\section{Materials and Methods}

2.1. GEPIA. GEPIA is a novel bioinformatics web server for analyzing RNA sequencing expression data across The Cancer Genome Atlas Program (TCGA) cancers [12]. TCGA is a landmark cancer genomics program that has molecularly characterized more than 20,000 primary cancers and matched normal samples spanning 33 cancer types. Tumor/normal differential expression analysis of JAKs in STAD was explored using the TCGA STAD dataset $(n=415)$ in GEPIA with analysis of variance (ANOVA). A $P$ value less than 0.05 indicated statistical significance.
2.2. UALCAN. UALCAN is designed for gene expression analysis, prognosis analysis, and methylation analysis based on the data from TCGA and Clinical Proteomic Tumor Analysis Consortium (CPTAC) [13]. In the current study, the correlation between JAK3 and TYK2 expression and the clinicopathological parameters of STAD patients, including the race, gender, age, $H$. pylori infection status, histological subtype, tumor grade, cancer stage, and nodal metastasis status of patients, were analyzed using the TCGA STAD dataset $(n=415)$. A $P$ value less than 0.05 indicated statistical significance.

2.3. The Kaplan-Meier Plotter (KM Plotter). The KM plotter is designed for the prognostic analysis of $54 \mathrm{k}$ genes (mRNA, miRNA, and protein) in certain types of cancers including breast, lung, and gastric cancer [14]. Here, the significance of JAK3 and TYK2 in determining the overall survival (OS), postprogression survival (PPS), and first progression (FP) of STAD was analyzed using the Kaplan-Meier curve. 

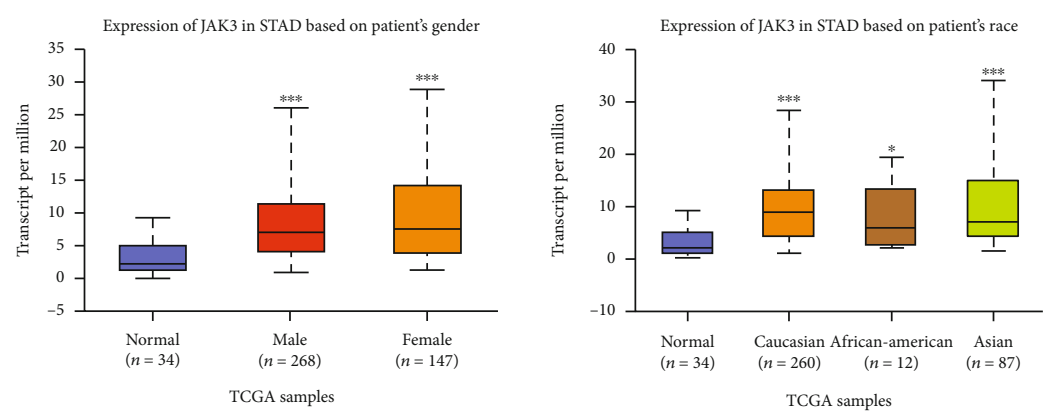

Expression of JAK3 in STAD based on H.pylori infection status
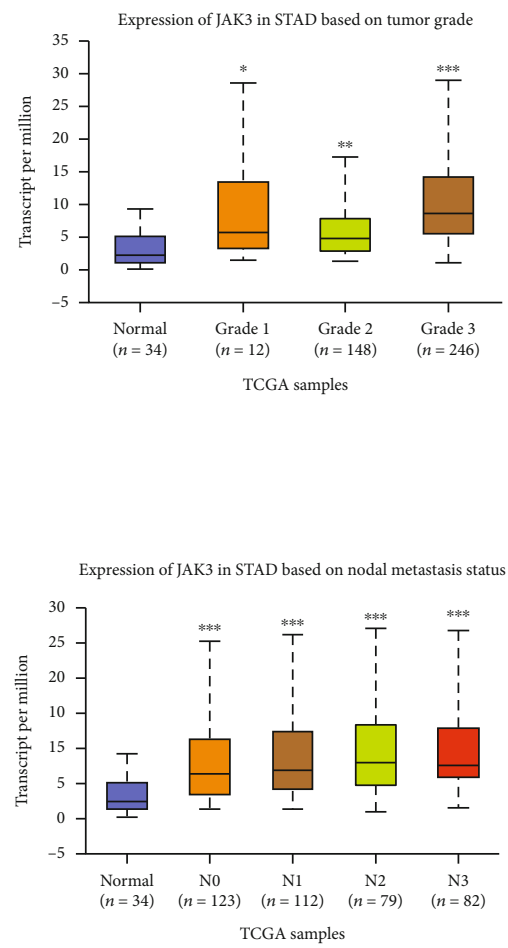
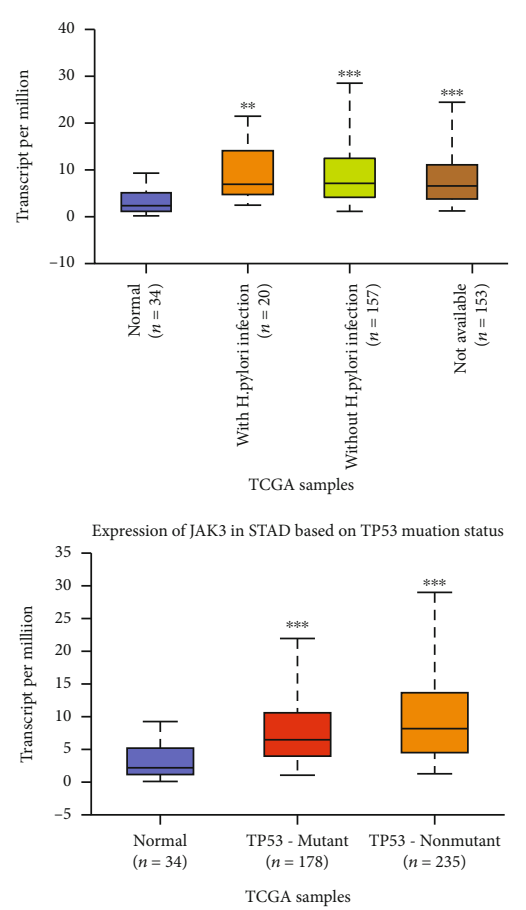

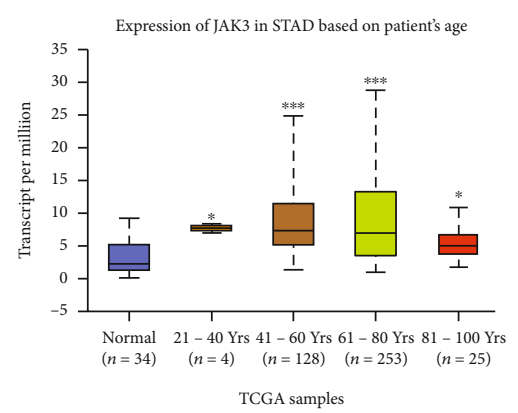

Expression of JAK3 in STAD based on histological subtypes

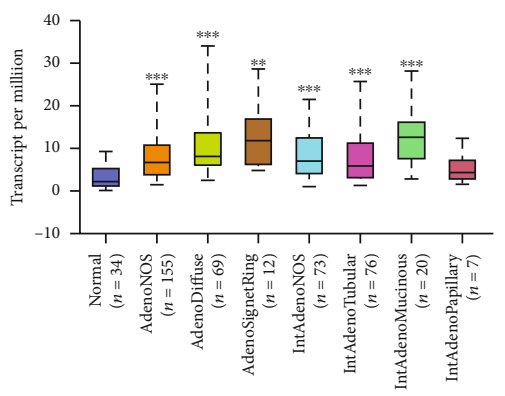

TCGA samples

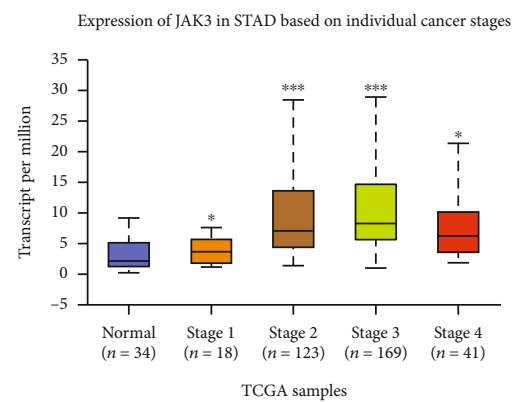

FIgURE 2: The expression of JAK3 in STAD in subgroup analyses (UALCAN). Subgroup analyses were performed based on patients' race, patients' gender, patients' age, H. pylori infection status, histological subtypes, tumor grade, individual cancer stages, and nodal metastasis status. STAD: stomach adenocarcinoma; ${ }^{*} P<0.05,{ }^{* *} P<0.01$, and ${ }^{* * *} P<0.001$.

The medium value of the JAK3 and TYK2 expressions was used to split patients into high-/low-expression groups.

2.4. cBioPortal. cBioPortal is a cancer genomics portal designed for exploring multidimensional cancer genomics data using the TCGA dataset [15]. We used cBioPortal to explore, visualize, and analyze the genetic alterations and mutations of JAK3 and TYK2 in STAD using the TCGA STAD dataset $(n=415)$. Furthermore, mRNA expression $z$ scores (RNA Seq V2 RSEM) were obtained ( $z$ score threshold, \pm 2.0 ). Protein expression $z$ scores (RPPA) were also obtained ( $z$ score threshold, \pm 2.0 ).

2.5. LinkedOmics. LinkedOmics is a bioinformatics web portal designed for accessing, analyzing, and comparing cancer multiomics data of various cancer types [16]. Complete data of 415 TCGA STAD patients were used to explore JAK3- and TYK2-associated genes via the Spearman correlation analysis. Moreover, Gene Set Enrich- ment Analysis (GSEA) was performed to explore JAK3and TYK2-associated functions (GO analysis and KEGG pathway analysis) in STAD, with the minimum number of genes being three and the $P$ value threshold being 0.05 . The transcription factor targets of JAK3 and TYK2 were also analyzed via GSEA.

2.6. TIMER. TIMER is a comprehensive resource for the systematic analysis of immune infiltrates across diverse cancer types [17]. In the current study, the Spearman correlation analysis was used to explore the correlation between the expression levels of JAK3/TYK2 and the abundance of immune cell infiltrates and the expression of gene biomarkers of immune cells [18-20]. The two-sided Wilcoxon rank-sum test was used to evaluate the effect of somatic copy number alterations (SCNAs) of JAK3/TYK2 on immune cell infiltrates. A $P$ value less than 0.05 indicated statistical significance. 

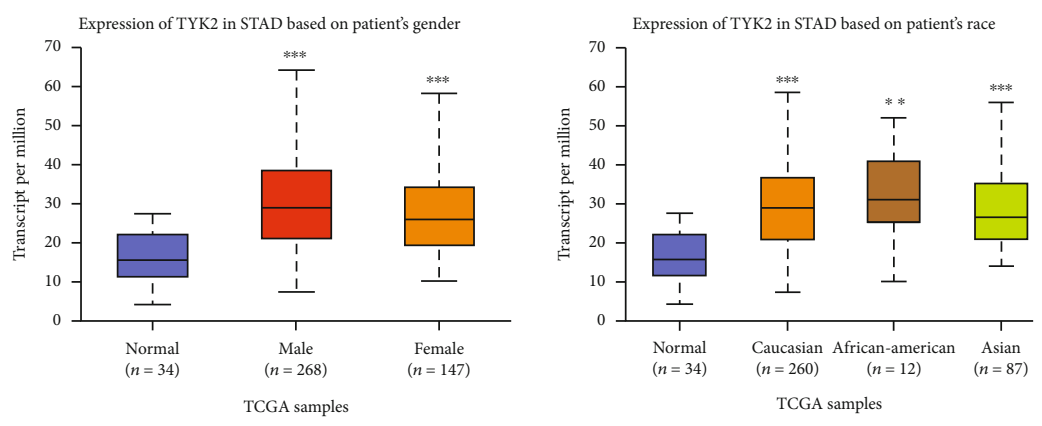

Expression of TYK2 in STAD based on H.pylori infection status
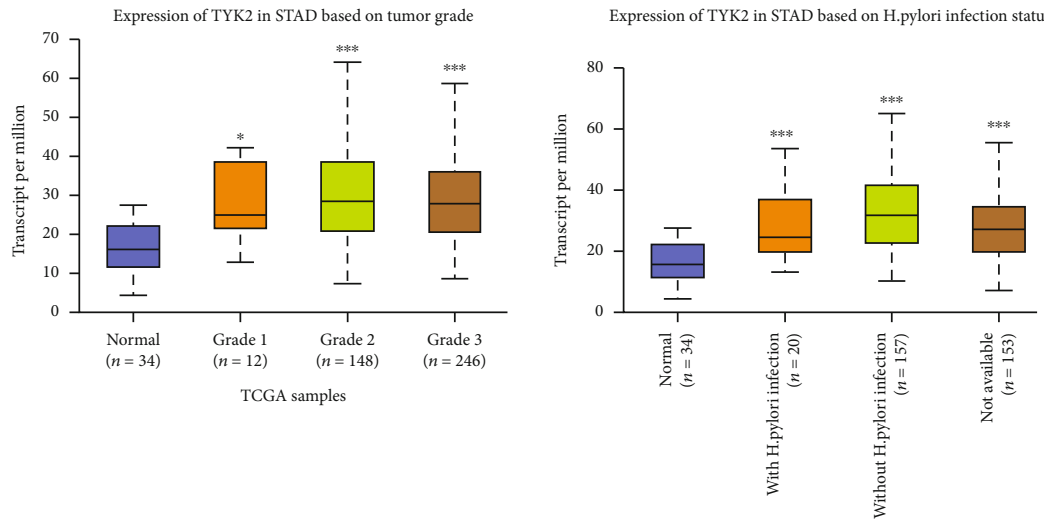

TCGA samples
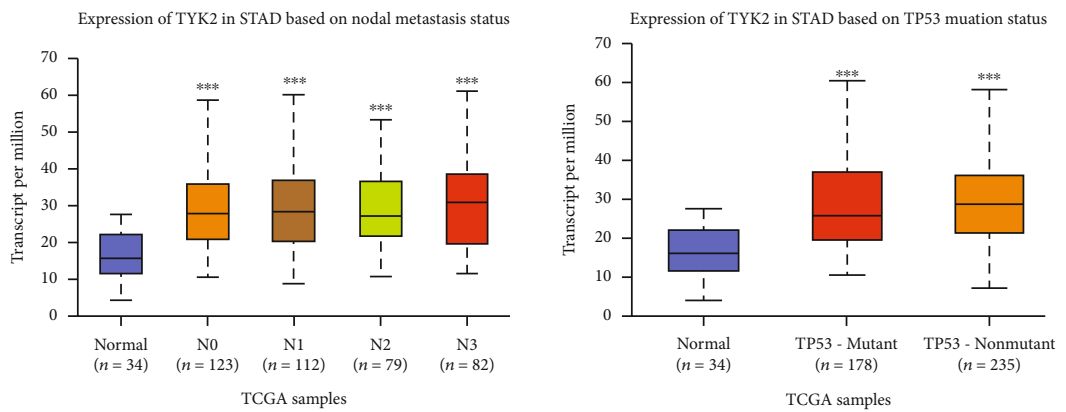

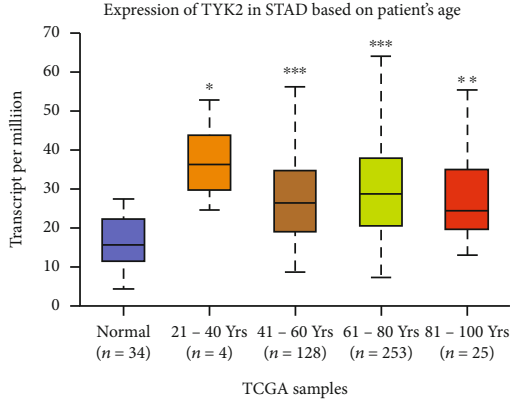

Expression of TYK2 in STAD based on histological subtypes

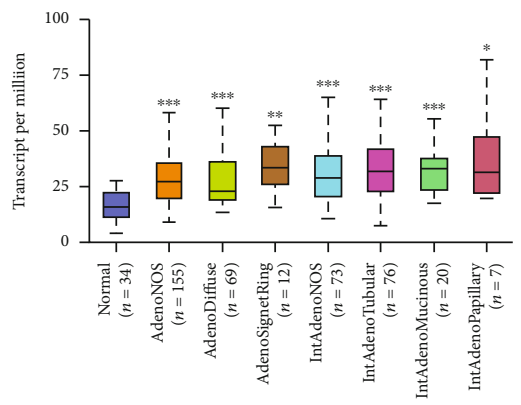

TCGA samples

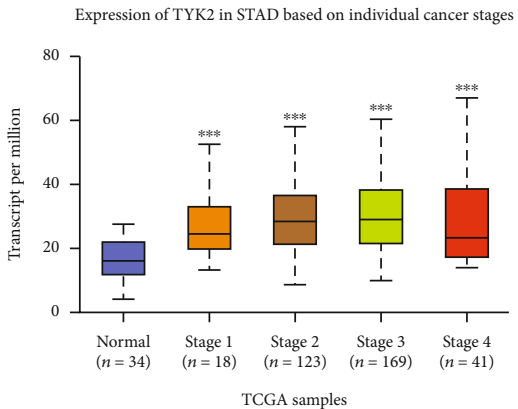

FIGURE 3: The expression of TYK2 in STAD in subgroup analyses (UALCAN). Subgroup analyses were performed based on patients' race, patients' gender, patients' age, $\mathrm{H}$. pylori infection status, histological subtypes, tumor grade, individual cancer stages, and nodal metastasis status. STAD: stomach adenocarcinoma; ${ }^{*} P<0.05,{ }^{* *} P<0.01$, and ${ }^{* * *} P<0.001$.

\section{Results}

3.1. JAK Expression in STAD. The level of JAKs in primary STAD was first determined using GEPIA. As shown in Figure 1, the expression levels of JAK3 (Figure 1(c), $P<0.05$ ) and TYK2 (Figure $1(\mathrm{~d}), \quad P<$ $0.05)$ were significantly elevated in STAD tissues compared with normal tissues. However, there was no difference in the expression levels of JAK1 (Figure 1(a)) and JAK2 (Figure 1(b)) between STAD tissues and normal tissues. We then analyzed the correlation between the expression levels of JAK3/TYK2 and the clinicopathological parameters of STAD patients. As expected, the mRNA levels of JAK3 were significantly increased in STAD during subgroup analyses based on the race, gender, age, H. pylori infection status, histological sub- type, tumor grade, cancer stage, and nodal metastasis status of patients (Figure 2). The same results were obtained for TYK2, and the mRNA levels of JAK3 were significantly increased in STAD during subgroup analyses based on the race, gender, age, $H$. pylori infection status, histological subtype, tumor grade, individual cancer stage, and nodal metastasis status of patients (Figure 3). Therefore, JAK3 and TYK2 may play a significant role in the tumorigenesis, progression, and aggressiveness of STAD.

3.2. JAK3/TYK2 As a Prognostic Biomarker in STAD. The prognostic value of JAK3/TYK2 in STAD was evaluated using the KM plotter. We found that STAD patients with high JAK3 levels had poor OS (HR = $1.45(1.22-1.71), P=2$ $\left.e^{-5}\right)$, FP $(\mathrm{HR}=1.41 \quad(1.15-1.72), \quad P=0.00076)$, and PPS 
OS

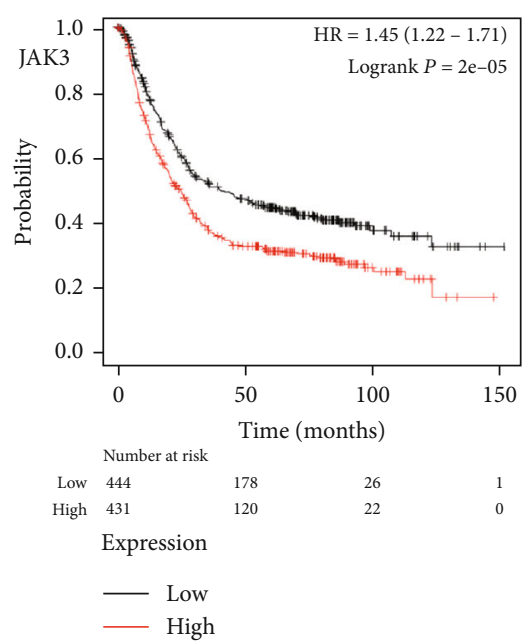

OS

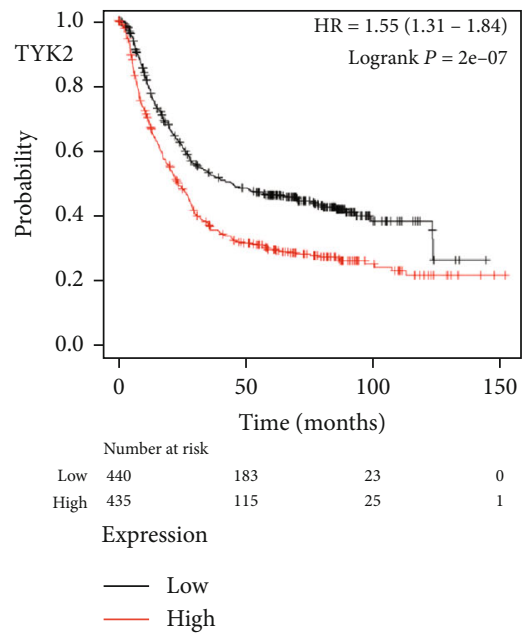

FP

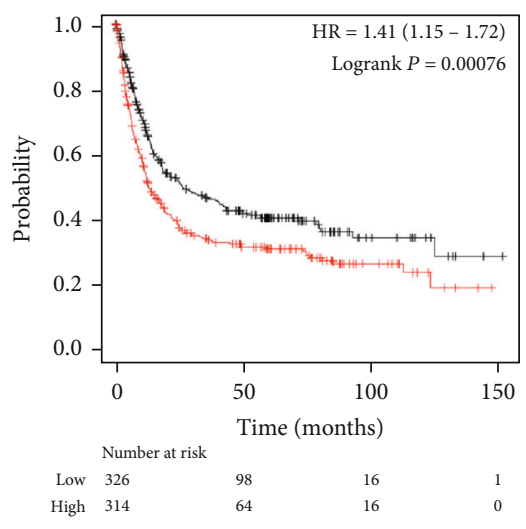

(a)

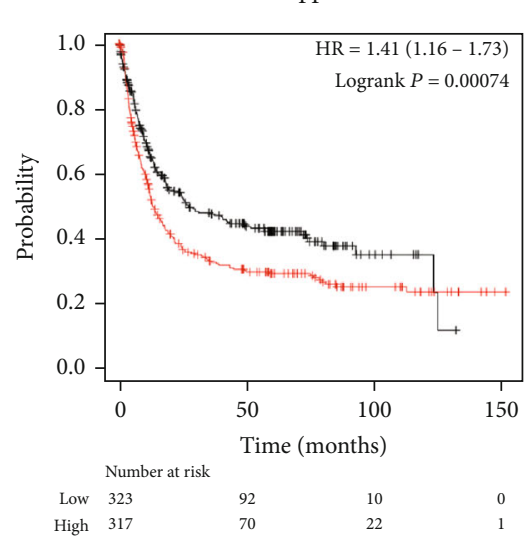

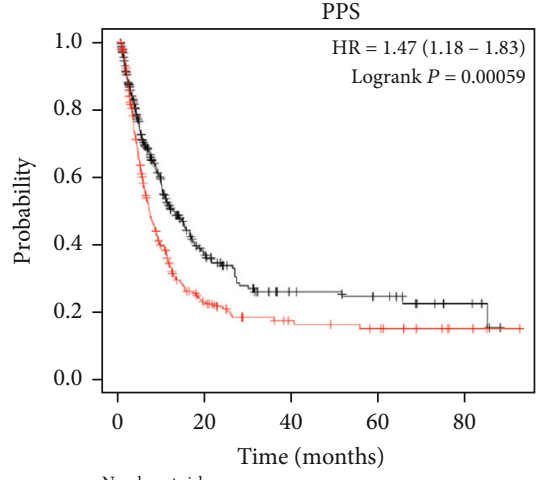

\begin{tabular}{rrrrrr}
\multicolumn{5}{c}{ Number at risk } \\
Low & 259 & 48 & 20 & 14 & 5 \\
High & 239 & 33 & 14 & 10 & 3
\end{tabular} 
TABLE 1: Correlation of JAK3/TYK2 mRNA expression and overall survival in STAD with different clinicopathological factors (Kaplan-Meier plotter).

\begin{tabular}{|c|c|c|c|c|c|c|}
\hline \multirow[b]{2}{*}{ Pathological parameters } & \multicolumn{6}{|c|}{ Overall survival } \\
\hline & $N$ & $\begin{array}{c}\text { JAK3 } \\
\text { Hazard radio }\end{array}$ & $P$ value & $N$ & $\begin{array}{c}\text { TYK2 } \\
\text { Hazard radio }\end{array}$ & $P$ value \\
\hline \multicolumn{7}{|l|}{ Sex } \\
\hline Female & 236 & $1.93(1.36-2.74)$ & 0.00017 & 236 & $1.5(1.06-2.14)$ & 0.023 \\
\hline Male & 544 & $1.59(1.23-2.04)$ & 0.00028 & 544 & $1.86(1.5-2.3)$ & $1 e^{-8}$ \\
\hline \multicolumn{7}{|l|}{ Stage } \\
\hline 1 & 67 & $3.61(1.35-9.65)$ & 0.0062 & 67 & $2.02(0.75-5.44)$ & 0.16 \\
\hline 2 & 140 & $2.39(1.3-4.38)$ & 0.0037 & 140 & $1.92(1.03-3.56)$ & 0.036 \\
\hline 3 & 305 & $1.56(0.17-2.09)$ & 0.0023 & 305 & $1.66(1.22-2.26)$ & 0.001 \\
\hline 4 & 148 & $0.76(0.49-1.17)$ & 0.21 & 148 & $0.71(0.48-1.06)$ & 0.091 \\
\hline \multicolumn{7}{|l|}{ Stage $\mathrm{T}$} \\
\hline 2 & 241 & $1.47(0.93-2.3)$ & 0.094 & 241 & $1.28(0.82-2)$ & 0.27 \\
\hline 3 & 204 & $0.78(0.54-1.13)$ & 0.19 & 204 & $1.47(0.97-2.22)$ & 0.065 \\
\hline 4 & 38 & $0.33(0.1-1.11)$ & 0.059 & 38 & $0.67(0.28-1.59)$ & 0.36 \\
\hline \multicolumn{7}{|l|}{ Stage N } \\
\hline 0 & 74 & $2.03(0.6-6.85)$ & 0.24 & 74 & $1.75(0.75-4.07)$ & 0.19 \\
\hline 1 & 225 & $2.63(1.49-4.66)$ & 0.00054 & 225 & $1.63(1.07-2.48)$ & 0.02 \\
\hline 2 & 121 & $0.72(0.45-1.15)$ & 0.17 & 121 & $0.64(0.41-1)$ & 0.048 \\
\hline 3 & 76 & $0.7(0.37-1.31)$ & 0.26 & 76 & $0.64(0.38-1.1)$ & 0.11 \\
\hline $1+2+3$ & 422 & $1.35(1.01-1.8)$ & 0.043 & 422 & $1.38(1.3-1.54)$ & 0.03 \\
\hline \multicolumn{7}{|l|}{ Stage $\mathrm{M}$} \\
\hline 0 & 444 & $1.3(0.97-1.75)$ & 0.082 & 444 & $1.32(1-1.76)$ & 0.053 \\
\hline 1 & 56 & $0.77(0.4-1.5)$ & 0.44 & 56 & $0.37(0.16-0.82)$ & 0.011 \\
\hline \multicolumn{7}{|l|}{ Lauren classification } \\
\hline Intestinal & 320 & $3.32(1.5-3.59)$ & 0.0001 & 320 & $1.74(1.27-2.39)$ & 0.00047 \\
\hline Diffuse & 241 & $1.38(0.97-1.97)$ & 0.07 & 241 & $1.15(0.81-1.62)$ & 0.44 \\
\hline \multicolumn{7}{|l|}{ Differentiation } \\
\hline Poor & 165 & $1.5(0.98-2.3)$ & 0.059 & 165 & $0.83(0.55-1.23)$ & 0.34 \\
\hline Moderate & 67 & $0.57(0.29-1.11)$ & 0.096 & 67 & $0.56(0.29-1.07)$ & 0.075 \\
\hline
\end{tabular}

3.3. Genetic Alterations of JAK3/TYK2 in STAD. cBioPortal was used to determine the genetic alterations of JAK3/TYK2 in STAD. We found that JAK3 and TYK2 were altered in $6 \%$ and $8 \%$ of all TCGA STAD cases, respectively (Figure 5(a)).

Genetic alterations of JAK3 and TYK2 in STAD comprised missense mutation, truncating mutation, amplification, deep deletion, high mRNA levels, and low mRNA levels. Thus, mutation is the most common type of JAK3/TYK2 genetic alteration. The mutation sites of JAK3/TYK2 in STAD are shown in Figures 5(b) and 5(c).

3.4. JAK3/TYK2 Correlated with Immune Infiltration in $S T A D$. An increasing number of studies have suggested an interaction between immune response and pathophysiological processes [21, 22]. Moreover, JAKs play a critical role in immune regulation by invoking intracellular signaling pathways in cancers [23]. Therefore, we next evaluated the correlation between JAK3/TYK2 and immune infiltration in
STAD. As shown in Figure 6, JAK3 levels showed a positive correlation with the abundance of CD8+ $\mathrm{T}$ cells (Cor $=0.521, P=3.87 e^{-27}$ ), CD4+ T cells (Cor $=0.509, P=$ $\left.1.52 e^{-25}\right)$, macrophages (Cor $\left.=0.332, P=5.33 e^{-12}\right)$, neutrophils (Cor $\left.=0.497, \quad P=1.62 e^{-24}\right)$, and dendritic cells $\left(\right.$ Cor $\left.=0.588, P=6.21 e^{-36}\right)$ (Figure 6(a)). We also found a positive correlation between TYK2 levels and the abundance of CD8+ T cells (Cor $=0.103, P=0.0468), C D 4+\mathrm{T}$ cells $\left(\right.$ Cor $\left.=0.249, P=1.44 e^{-06}\right)$, neutrophils (Cor $=0.129, P=$ $0.0127)$, and dendritic cells (Cor $=0.148, \quad P=0.00428)$ (Figure 6(b)). Interestingly, SCNA of JAK3/TYK2 could partially inhibit immune infiltration in STAD (Figures 6(c) and $6(\mathrm{~d}))$.

We also evaluated the correlation between JAK3/TYK2 and immune biomarkers in STAD. Previous studies have reported these biomarkers of immune cells [18-20]. As expected, the expression levels of JAK3/TYK 2 were positively correlated with the expression levels of immune biomarkers in STAD (Tables 4 and 5). We found that the expression 
TABLE 2: Correlation of JAK3/TYK2 mRNA expression and postprogression survival in STAD with different clinicopathological factors (Kaplan-Meier plotter).

\begin{tabular}{|c|c|c|c|c|c|c|}
\hline \multirow{3}{*}{ Pathological parameters } & \multicolumn{6}{|c|}{ Post progression survival } \\
\hline & & JAK3 & & & TYK2 & \\
\hline & $N$ & Hazard radio & $P$ value & $N$ & Hazard radio & $P$ value \\
\hline \multicolumn{7}{|l|}{ Sex } \\
\hline Female & 149 & $1.84(1.19-2.85)$ & 0.0053 & 149 & $2.36(1.49-3.73)$ & 0.00015 \\
\hline Male & 348 & $1.71(1.31-2.22)$ & 0.000055 & 348 & $2.32(1.79-3.02)$ & $1.1 e^{-10}$ \\
\hline \multicolumn{7}{|l|}{ Stage } \\
\hline 1 & 31 & $2.86(0.5-16.46)$ & 0.22 & 31 & $1.66(0.37-7.43)$ & 0.51 \\
\hline 2 & 105 & $2.5(1.28-4.87)$ & 0.0053 & 105 & $2.39(1.23-4.64)$ & 0.0081 \\
\hline 3 & 142 & $1.45(0.92-2.28)$ & 0.1 & 142 & $2.47(1.6-3.83)$ & $2.7 e^{-5}$ \\
\hline 4 & 104 & $1.66(1.01-2.72)$ & 0.045 & 104 & $0.68(0.42-1.11)$ & 0.12 \\
\hline \multicolumn{7}{|l|}{ Stage $\mathrm{T}$} \\
\hline 2 & 196 & $1.66(1.05-2.64)$ & 0.029 & 196 & $1.72(1.09-2.73)$ & 0.019 \\
\hline 3 & 150 & $1.21(0.8-1.82)$ & 0.36 & 150 & $1.91(1.17-3.12)$ & 0.0083 \\
\hline 4 & 29 & $0.45(0.15-1.38)$ & 0.15 & 29 & $0.61(0.22-1.64)$ & 0.32 \\
\hline \multicolumn{7}{|l|}{ Stage N } \\
\hline 0 & 41 & $2.59(0.72-9.35)$ & 0.13 & 41 & $2.57(0.77-8.57)$ & 0.11 \\
\hline 1 & 169 & $2.52(1.6-3.98)$ & 0.000039 & 169 & $2.48(1.58-3.91)$ & $4.8 e^{-5}$ \\
\hline 2 & 105 & $0.7(0.43-1.15)$ & 0.16 & 105 & $1.67(0.99-2.81)$ & 0.054 \\
\hline 3 & 63 & $1.65(0.86-3.18)$ & 0.13 & 63 & $0.53(0.29-0.95)$ & 0.032 \\
\hline $1+2+3$ & 337 & $1.44(1.06-1.95)$ & 0.02 & 337 & $1.62(1.21-2.17)$ & 0.0011 \\
\hline \multicolumn{7}{|l|}{ Stage M } \\
\hline 0 & 342 & $1.38(1-1.9)$ & 0.051 & 342 & $1.91(1.4-2.6)$ & $2.7 e^{-5}$ \\
\hline 1 & 36 & $2.35(1.08-5.13)$ & 0.028 & 36 & $0.73(0.33-1.63)$ & 0.44 \\
\hline \multicolumn{7}{|l|}{ Lauren classification } \\
\hline Intestinal & 192 & $1.69(1.08-2.66)$ & 0.02 & 192 & $1.86(1.22-2.84)$ & 0.0037 \\
\hline Diffuse & 176 & $1.5(0.99-2.26)$ & 0.053 & 176 & $1.55(1.05-2.29)$ & 0.028 \\
\hline \multicolumn{7}{|l|}{ Differentiation } \\
\hline Poor & 49 & $3.3(1.59-6.88)$ & 0.00076 & 49 & $1.68(0.82-3.41)$ & 0.15 \\
\hline Moderate & 24 & $2.06(0.75-5.63)$ & 0.15 & 24 & $0.63(0.26-1.56)$ & 0.32 \\
\hline
\end{tabular}

levels of biomarkers of CD8+ T cells (CD8A and CD8B), T cells (CD3D, CD3E, and CD2), B cells (CD19 and CD79A), monocytes (CD86 and CD115), and TAMs (CD68 and IL10) positively correlated with the expression levels of JAK3 and TYK2 in STAD. The expression levels of INOS, IRF5, CD163, VSIG4, MS4A4A, CD11b, and CCR7 were positively correlated with JAK3/TYK2 levels in STAD. All biomarkers of natural killer cells (KIR2DL1, KIR2DL3, KIR2DL4, KIR3DL1, KIR3DL2, KIR3DL3, and KIR2DS4) showed positive correlation with JAK3 expression. Similarly, all biomarkers of dendritic cells (KIR2DL1, KIR2DL3, KIR2DL4, KIR3DL1, KIR3DL2, KIR3DL3, and KIR2DS4), Th1 cells (TBX21, STAT4, STAT1, IIFNG, and TNF), Th2 cells (GATA3, STAT6, STAT5A, and IL13), and Tfh cells (BCL6 and IL21) showed a positive correlation with the JAK3 and TYK2 expressions. Moreover, levels of immune biomarkers of Treg cells (FOXP3, CCR8, and STAT5B) and $\mathrm{T}$ cell exhaustion (PD-1, CTLA4, LAG3, TIM-3, and GZMB) were positively associ- ated with JAK3 and TYK2 levels. These results indicate that JAK3 and TYK2 played a vital role in immune escape in the STAD microenvironment.

3.5. Enrichment Analysis of JAK3/TYK2 in STAD. The function module of LinkedOmics was used to performed enrichment analysis of JAK3/TYK2 in STAD. In all, 7855 genes (dark red dots) were positively correlated with JAK3, whereas 4687 genes (dark green dots) were negatively correlated with JAK3 in STAD (Supplementary Figure $2 \mathrm{~A}, P<0.05)$. Further, 50 significant gene sets that positively and negatively correlated with JAK3 in STAD are presented in Supplementary Figure 2A2C, respectively. Enrichment analysis performed via GSEA suggested that JAK3 is associated with adaptive immune response, protein transmembrane transport, DNA damage response, DNA damage detection, preribosomal structure, respiratory chain, cytokine binding, translation factor activity, RNA binding, snoRNA binding, and tRNA 
TABLE 3: Correlation of JAK3/TYK2 mRNA expression and first progression in STAD with different clinicopathological factors (KaplanMeier plotter).

\begin{tabular}{|c|c|c|c|c|c|c|}
\hline \multirow[b]{2}{*}{ Pathological parameters } & \multicolumn{6}{|c|}{ First progression } \\
\hline & $N$ & $\begin{array}{c}\text { JAK3 } \\
\text { Hazard radio }\end{array}$ & $P$ value & $N$ & $\begin{array}{c}\text { TYK2 } \\
\text { Hazard radio }\end{array}$ & $P$ value \\
\hline \multicolumn{7}{|l|}{ Sex } \\
\hline Female & 201 & $2.02(1.38-2.95)$ & 0.00021 & 201 & $1.37(0.94-2)$ & 0.097 \\
\hline Male & 437 & $1.42(1.1-1.84)$ & 0.0076 & 437 & $2.07(1.6-2.68)$ & $1.4 e^{-8}$ \\
\hline \multicolumn{7}{|l|}{ Stage } \\
\hline 1 & 60 & $2.37(0.79-7.1)$ & 0.11 & 60 & $0.54(0.18-1.68)$ & 0.28 \\
\hline 2 & 131 & $1.5(0.79-2.84)$ & 0.21 & 131 & $1.42(0.77-2.61)$ & 0.25 \\
\hline 3 & 186 & $1.52(1.04-2.24)$ & 0.031 & 186 & $1.36(0.94-1.97)$ & 0.1 \\
\hline 4 & 141 & $0.64(0.41-1.02)$ & 0.057 & 141 & $0.73(0.48-1.11)$ & 0.14 \\
\hline \multicolumn{7}{|l|}{ Stage $\mathrm{T}$} \\
\hline 2 & 239 & $1.51(0.92-2.45)$ & 0.097 & 239 & $1.21(0.8-1.83)$ & 0.36 \\
\hline 3 & 204 & $0.7(0.49-1.01)$ & 0.054 & 204 & $1.35(0.9-2.02)$ & 0.15 \\
\hline 4 & 39 & $0.46(0.2-1.09)$ & 0.072 & 39 & $0.77(0.36-1.66)$ & 0.5 \\
\hline \multicolumn{7}{|l|}{ Stage N } \\
\hline 0 & 72 & $2.22(0.66-7.49)$ & 0.19 & 72 & $1.56(0.66-3.65)$ & 0.3 \\
\hline 1 & 222 & $2.27(1.34-3.82)$ & 0.0016 & 222 & $1.45(0.98-2.15)$ & 0.059 \\
\hline 2 & 125 & $0.84(0.53-1.32)$ & 0.44 & 125 & $0.59(0.38-0.91)$ & 0.015 \\
\hline 3 & 76 & $1.31(0.72-2.37)$ & 0.37 & 76 & $0.73(0.41-1.33)$ & 0.3 \\
\hline $1+2+3$ & 423 & $1.32(1-1.74)$ & 0.049 & 423 & $0.89(0.67-1.17)$ & 0.4 \\
\hline \multicolumn{7}{|l|}{ Stage $\mathrm{M}$} \\
\hline 0 & 443 & $1.35(0.98-1.85)$ & 0.066 & 443 & $1.21(0.92-1.59)$ & 0.17 \\
\hline 1 & 56 & $0.6(0.33-1.11)$ & 0.099 & 56 & $0.41(0.19-0.92)$ & 0.026 \\
\hline \multicolumn{7}{|l|}{ Lauren classification } \\
\hline Intestinal & 263 & $1.74(1.15-2.62)$ & 0.0078 & 263 & $1.28(0.88-1.88)$ & 0.2 \\
\hline Diffuse & 231 & $1.25(0.87-1.79)$ & 0.22 & 231 & $0.85(0.59-1.24)$ & 0.4 \\
\hline \multicolumn{7}{|l|}{ Differentiation } \\
\hline Poor & 121 & $1.32(0.84-2.09)$ & 0.23 & 121 & $0.67(0.41-1.08)$ & 0.095 \\
\hline Moderate & 67 & $0.63(0.33-1.19)$ & 0.15 & 67 & $0.6(0.32-1.13)$ & 0.11 \\
\hline
\end{tabular}

binding during GO analysis (Supplementary Figure 2D-2F). Moreover, KEGG analysis revealed that JAK3 was involved in cytokine-cytokine receptor interactions, chemokine signaling pathway, NF-kappa B signaling pathway, Th17 cell differentiation, and $\mathrm{T}$ cell receptor signaling pathway and that JAK3 was associated with cell adhesion molecules (CAMs) (Supplementary Figure 2G and Supplementary Figure 3).

The results of enrichment analysis of TYK2 in STAD are shown Supplementary Figure 4. We found that 5756 genes (dark red dots) were positively correlated with TYK2, whereas 3993 genes (dark green dots) were negatively correlated with TYK2 in STAD (Supplementary Figure 4A, $P<0.05)$. Further, 50 significant gene sets that positively and negatively correlated with TYK2 in STAD are presented in Supplementary Figure 4B and 4C, respectively. Enrichment analysis performed by GSEA suggested that TYK2 was associated with the regulation of leukocyte activation, adaptive immune responses, translational initiation, mitochondrial matrix, ribosomal structure, translation factor activity, cytokine receptor activity, rRNA binding, and protein transporter activity during GO analysis (Supplementary Figure 4D-4F). Furthermore, KEGG analysis revealed that JAK3 was associated with ribosomal structure, cytokine-cytokine receptor interaction, JAK-STAT signaling pathway, RNA transport, CAMs, and Th1 and Th2 cell differentiation (Supplementary Figure 4G and Supplementary Figure 5).

\section{Discussion}

Increasing evidence has revealed that JAKs play an important role in the regulation of cytokine signaling, thus affecting basic cellular mechanisms, such as cell invasion, proliferation, apoptosis, and cellular immunity [5, 24]. Moreover, JAK-associated signaling pathways are associated with tumorigenesis and progression of cancers, including lung cancer, renal cell carcinoma, and lung cancer [25-27]. However, specific functions of the JAK family in STAD remain to be systematically described. Therefore, our study was conducted to clarify the role of JAKs in STAD. 


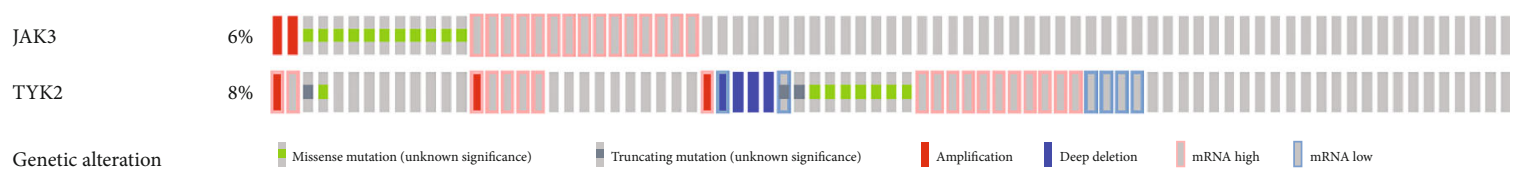

(a)

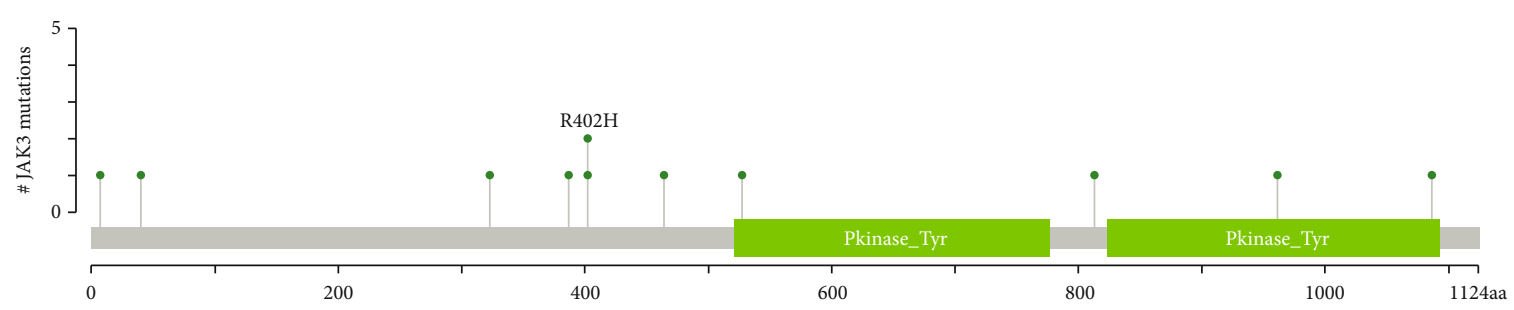

(b)

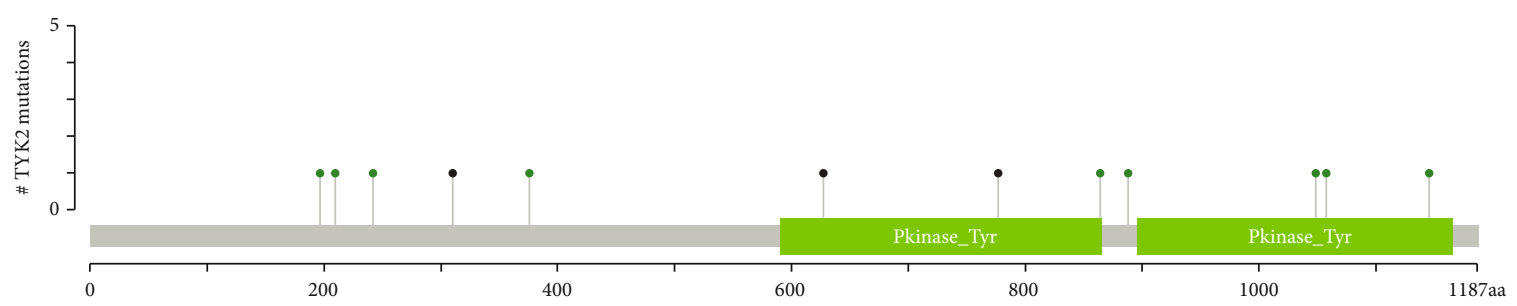

(c)

FIGURE 5: Genetic alteration of JAK3/TYK2 in STAD (cBioPortal). (a) OncoPrint of JAK3/TYK2 alterations in STAD. (b, c) Mutation sites of JAK3/TYK2 in STAD.

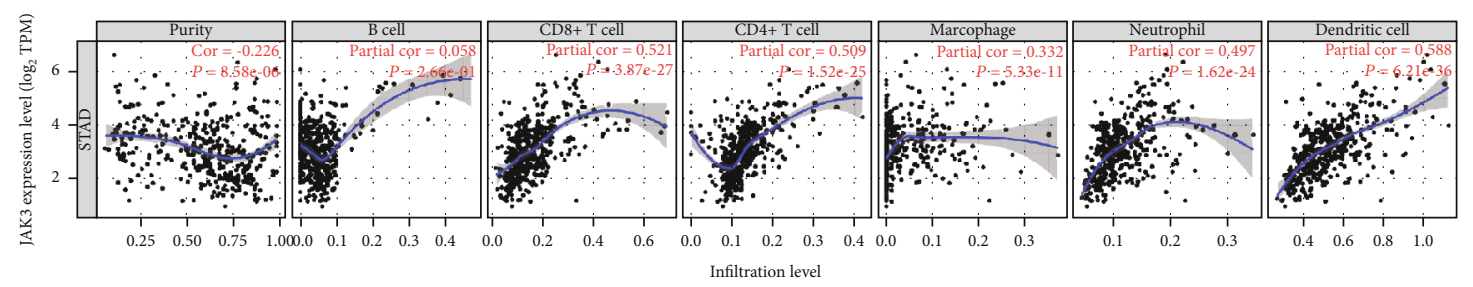

(a)

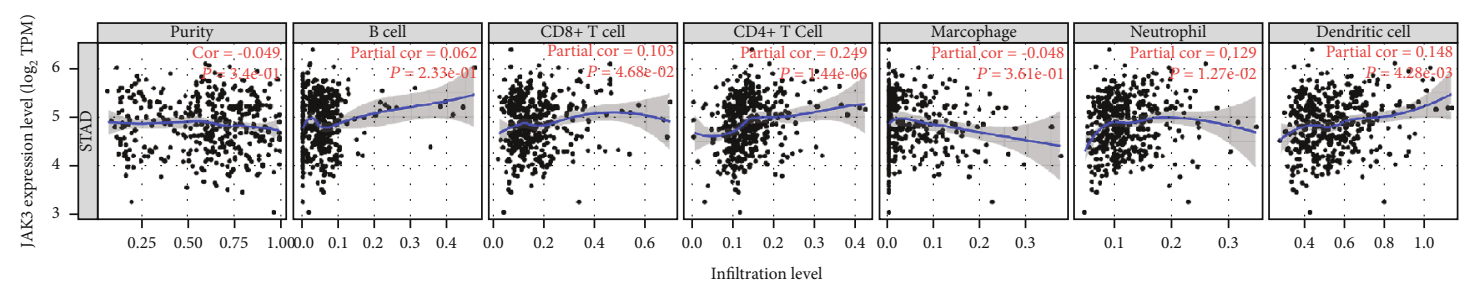

(b)

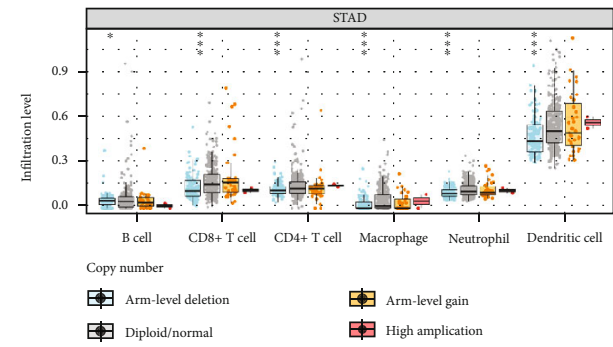

(c)

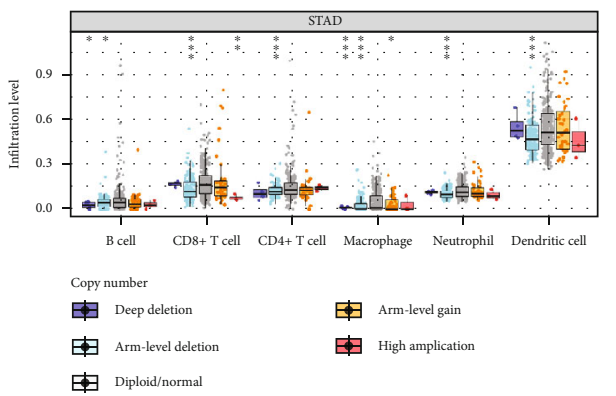

(d)

FIGURE 6: The correlation between JAK3/TYK2 and immune infiltration (TIMER). (Aa, b) The correlation between JAK3/TYK2 expression and the abundance of CD8+ T cells, CD4+ T cells, macrophage, neutrophils, and dendritic cells. (c, d) The correlation between SCNA of JAK3/TYK2 and immune cell infiltration. SCNA: somatic copy number alterations; ${ }^{*} P<0.05,{ }^{* *} P<0.01$, and ${ }^{* * *} P<0.001$. 
TABLE 4: Correlation analysis between JAK3 and gene biomarkers of immune cells in STAD (TIMER).

\begin{tabular}{|c|c|c|c|c|c|}
\hline \multirow{3}{*}{ Description } & \multirow{3}{*}{ Biomarkers } & \multicolumn{4}{|c|}{ STAD } \\
\hline & & \multicolumn{2}{|c|}{ None } & \multicolumn{2}{|c|}{ Purity } \\
\hline & & Cor & $P$ value & Cor & $P$ value \\
\hline \multirow{2}{*}{ CD8+ T cell } & CD8A & 0.7 & $* * *$ & 0.684 & $* * *$ \\
\hline & CD8B & 0.553 & $* * *$ & 0.539 & $* * *$ \\
\hline \multirow{3}{*}{$\mathrm{T}$ cell (general) } & CD3D & 0.711 & $* * *$ & 0.696 & $* * *$ \\
\hline & CD3E & 0.735 & $* * *$ & 0.73 & $* * *$ \\
\hline & $\mathrm{CD} 2$ & 0.701 & $* * *$ & 0.685 & $* * *$ \\
\hline \multirow{2}{*}{ B cell } & CD19 & 0.658 & $* * *$ & 0.648 & $* * *$ \\
\hline & CD79A & 0.629 & $* * *$ & 0.605 & $* * *$ \\
\hline \multirow[b]{2}{*}{ Monocyte } & CD86 & 0.562 & $* * *$ & 0.536 & $* * *$ \\
\hline & CD115(CSF1R) & 0.541 & $* * *$ & 0.527 & $* * *$ \\
\hline \multirow{3}{*}{ TAM } & CCL2 & 0.441 & $* * *$ & 0.404 & $* * *$ \\
\hline & CD68 & 0.318 & $* * *$ & 0.294 & $* * *$ \\
\hline & IL10 & 0.482 & $* * *$ & 0.45 & $* * *$ \\
\hline \multirow{3}{*}{ M1 macrophage } & INOS (NOS2) & 0.135 & ** & 0.129 & * \\
\hline & IRF5 & 0.401 & $* * *$ & 0.378 & $* * *$ \\
\hline & COX2(PTGS2) & 0.036 & 0.465 & 0.006 & 0.915 \\
\hline \multirow{3}{*}{ M2 macrophage } & CD163 & 0.482 & $* * *$ & 0.466 & $* * *$ \\
\hline & VSIG4 & 0.389 & $* * *$ & 0.382 & $* * *$ \\
\hline & MS4A4A & 0.474 & $* * *$ & 0.451 & $* * *$ \\
\hline \multirow{3}{*}{ Neutrophils } & CD66b (CEACAM8) & 0.054 & 0.269 & 0.053 & 0.307 \\
\hline & CD11b (ITGAM) & 0.563 & $* * *$ & 0.554 & $* * *$ \\
\hline & CCR7 & 0.725 & $* * *$ & 0.708 & $* * *$ \\
\hline \multirow{7}{*}{ Natural killer cell } & KIR2DL1 & 0.283 & $* *$ & 0.261 & $* * *$ \\
\hline & KIR2DL3 & 0.264 & $* * *$ & 0.217 & $* * *$ \\
\hline & KIR2DL4 & 0.301 & $* * *$ & 0.268 & $* * *$ \\
\hline & KIR3DL1 & 0.29 & $* * *$ & 0.268 & $* * *$ \\
\hline & KIR3DL2 & 0.433 & $* * *$ & 0.396 & $* * *$ \\
\hline & KIR3DL3 & 0.103 & $*$ & 0.103 & * \\
\hline & KIR2DS4 & 0.283 & $* *$ & 0.253 & $* * *$ \\
\hline \multirow{7}{*}{ Dendritic cell } & HLA-DPB1 & 0.581 & $* * *$ & 0.556 & $* * *$ \\
\hline & HLA-DQB1 & 0.466 & $* * *$ & 0.418 & $* * *$ \\
\hline & HLA-DRA & 0.496 & $* * *$ & 0.471 & $* * *$ \\
\hline & HLA-DPA1 & 0.495 & $* * *$ & 0.464 & $* * *$ \\
\hline & BDCA-1(CD1C) & 0.52 & $* * *$ & 0.482 & $* * *$ \\
\hline & BDCA-4(NRP1) & 0.472 & $* * *$ & 0.454 & $* * *$ \\
\hline & CD11c (ITGAX) & 0.64 & $* * *$ & 0.625 & $* * *$ \\
\hline \multirow{5}{*}{ Th1 } & T-bet (TBX21) & 0.753 & $* * *$ & 0.753 & *** \\
\hline & STAT4 & 0.759 & $* * *$ & 0.757 & $* * *$ \\
\hline & STAT1 & 0.466 & $* * *$ & 0.487 & $* * *$ \\
\hline & IFN-g (IFNG) & 0.438 & $* * *$ & 0.429 & $* * *$ \\
\hline & TNF-a (TNF) & 0.361 & $* * *$ & 0.322 & $* * *$ \\
\hline
\end{tabular}


TABLE 4: Continued.

\begin{tabular}{|c|c|c|c|c|c|}
\hline \multirow{3}{*}{ Description } & \multirow{3}{*}{ Biomarkers } & \multicolumn{4}{|c|}{ STAD } \\
\hline & & \multicolumn{2}{|c|}{ None } & \multicolumn{2}{|c|}{ Purity } \\
\hline & & Cor & $P$ value & Cor & $P$ value \\
\hline \multirow{4}{*}{ Th2 } & GATA3 & 0.633 & $* * *$ & 0.625 & *** \\
\hline & STAT6 & 0.296 & $* * *$ & 0.321 & *** \\
\hline & STAT5A & 0.581 & $* * *$ & 0.576 & $* * *$ \\
\hline & IL13 & 0.214 & $* * *$ & 0.218 & $* * *$ \\
\hline \multirow{2}{*}{ Tfh } & BCL6 & 0.426 & $* * *$ & 0.413 & $* * *$ \\
\hline & IL21 & 0.384 & $* * *$ & 0.362 & $* * *$ \\
\hline \multirow{2}{*}{ Th17 } & STAT3 & 0.455 & $* * *$ & 0.467 & $* * *$ \\
\hline & IL17A & 0.075 & 0.12 & 0.087 & 0.0919 \\
\hline \multirow{4}{*}{ Treg } & FOXP3 & 0.711 & $* * *$ & 0.679 & $* * *$ \\
\hline & CCR8 & 0.657 & $* * *$ & 0.644 & $* * *$ \\
\hline & STAT5B & 0.534 & $* * *$ & 0.545 & $* * *$ \\
\hline & TGFb (TGFB1) & 0.536 & $* * *$ & 0.52 & $* * *$ \\
\hline \multirow{5}{*}{$\mathrm{T}$ cell exhaustion } & PD-1 (PDCD1) & 0.725 & $* * *$ & 0.718 & $* * *$ \\
\hline & CTLA4 & 0.645 & $* * *$ & 0.623 & $* * *$ \\
\hline & LAG3 & 0.608 & $* * *$ & 0.597 & $* * *$ \\
\hline & TIM-3 (HAVCR2) & 0.567 & $* * *$ & 0.545 & $* * *$ \\
\hline & GZMB & 0.449 & $* * *$ & 0.407 & $* * *$ \\
\hline
\end{tabular}

${ }^{*} P<0.05,{ }^{* *} P<0.001$, and ${ }^{* * *} P<0.001$.

In this study, we found that the expression levels of JAK3 and TYK2 were higher in tumor tissues than in normal tissues in STAD. Further analysis revealed that JAK3 and TYK2 served as prognostic biomarkers in STAD and were associated with tumorigenesis, progression, and metastasis of STAD. Previous studies have also suggested that JAKs serve as biomarkers in certain types of cancers. In clear cell renal cell carcinoma, JAK3 acted as a novel biomarker and was associated with immune infiltration [26]. Another study revealed that JAK2 was a prognostic biomarker in skin cutaneous melanoma and was involved in gene regulation [10]. Moreover, JAK2 and TYK2 were suggested to be potential biomarkers for the diagnosis of hepatocellular carcinoma.

Another significant finding of our study is that JAK3 and TYK2 were associated with the abundance of immune cells, including CD8+ T cells, CD4+ T cells, neutrophils, and dendritic cells. Moreover, the expression levels of JAK3/TYK2 were positively correlated with the expression levels of immune biomarkers in STAD, demonstrating that JAK3 and TYK2 may play a vital role in immune escape in the STAD microenvironment. Previous studies have also clarified the significant role of JAK3 and TYK2 in the tumor microenvironment and immune response. JAK3 has been reported to be involved in hematopoiesis during $\mathrm{T}$ cell development by mediating innate and adaptive immunityassociated signaling [28]. Another study has reported that JAK3 deficiency can inhibit the development of innate lymphoid cells [29]. In lung cancer, JAK3 variants can promote PD-L1 induction in the tumor immune microenvironment and JAK3 activation may contribute to the long-term efficacy of PD-L1 [30]. A CTLA-4-TYK2STAT3 axis has been reported in B cell lymphoma cells and tumor-associated B cells and is relevant to immune checkpoint therapy [31].

In this study, enrichment analysis was performed, which revealed the functions and pathways of JAK3 and TYK2 in STAD, indicating that JAK3 and TYK2 were mainly associated with adaptive immune responses, translational initiations, DNA damage responses, chemokine signaling pathway, NF-kappa B signaling pathway, ribosomal structure, and JAK-STAT signaling pathway. It is well known that NF-kappa B signaling pathway is involved in inflammation and innate immunity and plays a vital role in cancer initiation and progression [32]. Moreover, NF- $\kappa \mathrm{B}$ suppression can inhibit tumor cell growth and promote cell apoptosis in cholangiocarcinoma [33]. Increasing evidence has also highlighted the significant role of JAK/STAT/NF- $\kappa \mathrm{B}$ signaling pathway in the immune response, axial spondyloarthritis, type 2 diabetes, metabolic disorders, and cancers [34-38]. Thus, JAK3 and TYK2 may exert functions in STAD via JAK-STAT and NF- $\kappa$ B signaling pathway.

This study has some limitations. First, in our study, we performed analysis at an mRNA level; it would be better to verify our results at a protein level. Furthermore, validation of our results by performing in vivo and in vitro experiments is warranted. 
TABLE 5: Correlation analysis between TYK2 and gene biomarkers of immune cells in STAD (TIMER).

\begin{tabular}{|c|c|c|c|c|c|}
\hline \multirow{3}{*}{ Description } & \multirow{3}{*}{ Biomarkers } & \multicolumn{4}{|c|}{ STAD } \\
\hline & & \multicolumn{2}{|c|}{ None } & \multicolumn{2}{|c|}{ Purity } \\
\hline & & Cor & $P$ value & Cor & $P$ value \\
\hline \multirow{2}{*}{ CD8+ T cell } & CD8A & 0.298 & $* * *$ & 0.318 & *** \\
\hline & CD8B & 0.161 & ** & 0.166 & $* *$ \\
\hline \multirow{3}{*}{$\mathrm{T}$ cell (general) } & CD3D & 0.214 & $* * *$ & 0.233 & $* * *$ \\
\hline & CD3E & 0.295 & $* * *$ & 0.32 & $* * *$ \\
\hline & $\mathrm{CD} 2$ & 0.257 & $* * *$ & 0.278 & $* * *$ \\
\hline \multirow{2}{*}{ B cell } & CD19 & 0.296 & $* * *$ & 0.309 & $* * *$ \\
\hline & CD79A & 0.231 & $* * *$ & 0.242 & $* * *$ \\
\hline \multirow{2}{*}{ Monocyte } & CD86 & 0.213 & $* * *$ & 0.219 & $* * *$ \\
\hline & CD115(CSF1R) & 0.302 & $* * *$ & 0.296 & $* * *$ \\
\hline \multirow{3}{*}{ TAM } & CCL2 & 0.039 & 0.432 & 0.028 & 0.586 \\
\hline & CD68 & 0.267 & $* * *$ & 0.265 & $* * *$ \\
\hline & IL10 & 0.288 & $* * *$ & 0.286 & $* * *$ \\
\hline \multirow{3}{*}{ M1 macrophage } & INOS (NOS2) & 0.17 & $* * *$ & 0.165 & $* *$ \\
\hline & IRF5 & 0.37 & $* * *$ & 0.363 & $* * *$ \\
\hline & COX2(PTGS2) & 0.009 & 0.858 & 0 & 1 \\
\hline \multirow{3}{*}{ M2 macrophage } & CD163 & 0.31 & $* * *$ & 0.304 & $* * *$ \\
\hline & VSIG4 & 0.159 & $* *$ & 0.152 & $* *$ \\
\hline & MS4A4A & 0.18 & $* * *$ & 0.174 & $* * *$ \\
\hline \multirow{3}{*}{ Neutrophils } & CD66b (CEACAM8) & 0.018 & 0.713 & 0.028 & 0.591 \\
\hline & CD11b (ITGAM) & 0.411 & $* * *$ & 0.413 & $* * *$ \\
\hline & CCR7 & 0.31 & $* * *$ & 0.331 & $* * *$ \\
\hline \multirow{7}{*}{ Natural killer cell } & KIR2DL1 & 0.036 & 0.463 & 0.055 & 0.283 \\
\hline & KIR2DL3 & 0.037 & 0.448 & 0.046 & 0.372 \\
\hline & KIR2DL4 & 0.158 & $* *$ & 0.178 & $* * *$ \\
\hline & KIR3DL1 & 0.114 & 0.02 & 0.128 & 0.0126 \\
\hline & KIR3DL2 & 0.145 & $* *$ & 0.151 & $* *$ \\
\hline & KIR3DL3 & 0.094 & 0.0554 & 0.114 & * \\
\hline & KIR2DS4 & 0.09 & 0.0673 & 0.104 & $*$ \\
\hline \multirow{7}{*}{ Dendritic cell } & HLA-DPB1 & 0.26 & $* * *$ & 0.274 & $* * *$ \\
\hline & HLA-DQB1 & 0.249 & $* * *$ & 0.271 & $* * *$ \\
\hline & HLA-DRA & 0.263 & $* * *$ & 0.278 & $* * *$ \\
\hline & HLA-DPA1 & 0.259 & $* * *$ & 0.269 & $* * *$ \\
\hline & BDCA-1(CD1C) & 0.175 & $* * *$ & 0.159 & $* *$ \\
\hline & BDCA-4(NRP1) & 0.26 & $* * *$ & 0.244 & $* * *$ \\
\hline & CD11c (ITGAX) & 0.393 & $* * *$ & 0.404 & $* * *$ \\
\hline \multirow{5}{*}{ Th1 } & T-bet (TBX21) & 0.372 & $* * *$ & 0.397 & $* * *$ \\
\hline & STAT4 & 0.31 & $* * *$ & 0.33 & $* * *$ \\
\hline & STAT1 & 0.373 & $* * *$ & 0.393 & $* * *$ \\
\hline & IFN-g (IFNG) & 0.24 & $* * *$ & 0.265 & $* * *$ \\
\hline & TNF-a (TNF) & 0.254 & $* * *$ & 0.255 & $* * *$ \\
\hline
\end{tabular}


TABle 5: Continued.

\begin{tabular}{|c|c|c|c|c|c|}
\hline \multirow{3}{*}{ Description } & \multirow{3}{*}{ Biomarkers } & \multicolumn{4}{|c|}{ STAD } \\
\hline & & \multicolumn{2}{|c|}{ None } & \multicolumn{2}{|c|}{ Purity } \\
\hline & & Cor & $P$ value & Cor & $P$ value \\
\hline \multirow{4}{*}{ Th2 } & GATA3 & 0.218 & $* * *$ & 0.235 & *** \\
\hline & STAT6 & 0.435 & $* * *$ & 0.438 & $* * *$ \\
\hline & STAT5A & 0.546 & $* * *$ & 0.561 & $* * *$ \\
\hline & IL13 & 0.101 & 0.039 & 0.118 & 0.0213 \\
\hline \multirow{2}{*}{ Tfh } & BCL6 & 0.232 & $* * *$ & 0.233 & $* * *$ \\
\hline & IL21 & 0.216 & $* * *$ & 0.211 & $* * *$ \\
\hline \multirow{2}{*}{ Th17 } & STAT3 & 0.457 & $* * *$ & 0.456 & $* * *$ \\
\hline & IL17A & 0.057 & 0.25 & 0.068 & 0.189 \\
\hline \multirow{4}{*}{ Treg } & FOXP3 & 0.439 & $* * *$ & 0.467 & *** \\
\hline & CCR8 & 0.372 & $* * *$ & 0.379 & $* * *$ \\
\hline & STAT5B & 0.493 & $* * *$ & 0.489 & $* * *$ \\
\hline & TGFb (TGFB1) & 0.288 & $* * *$ & 0.293 & $* * *$ \\
\hline \multirow{5}{*}{$\mathrm{T}$ cell exhaustion } & PD-1 (PDCD1) & 0.411 & $* * *$ & 0.448 & **** \\
\hline & CTLA4 & 0.322 & $* * *$ & 0.347 & $* * *$ \\
\hline & LAG3 & 0.27 & $* * *$ & 0.296 & $* * *$ \\
\hline & TIM-3 (HAVCR2) & 0.306 & $* * *$ & 0.314 & $* * *$ \\
\hline & GZMB & 0.159 & $* *$ & 0.174 & $* * *$ \\
\hline
\end{tabular}

${ }^{*} P<0.05,{ }^{* *} P<0.001$, and ${ }^{* * *} P<0.001$.

In conclusion, our results demonstrated that JAK3 and TYK2 serve as prognostic biomarkers and are associated with immune infiltration in STAD, providing additional data about biomarkers, STAD prognosis, and therapy.

\section{Data Availability}

The analyzed data sets generated during the study are available from the corresponding author on reasonable request.

\section{Conflicts of Interest}

The authors declare that they have no competing interests.

\section{Authors' Contributions}

Lingkai Meng and Wang Li were responsible for the design of the study and writing the manuscript. Ling Ding and Yue Yu were responsible for data analysis work. All authors read and approved the final manuscript.

\section{Acknowledgments}

This study was funded by the Scientific Research Funding of Tianjin Medical University Chu Hsien-I Memorial Hospital (account number 2018ZDKF03, 2018ZDKF04).

\section{Supplementary Materials}

Supplementary Figure 1 The expression of JAKs in STAD (UALCAN). The expressions of JAK1, JAK2, JAK3, and TYK2 were significantly elevated in STAD tissues at mRNA level. STAD: stomach adenocarcinoma; ${ }^{* * *} P<0.001$. Supplementary Figure 2. The enrichment analysis of JAK3 in STAD (LinkedOmics). (A) A Pearson test was used to analyze correlations between JAK3 and genes differentially expressed in STAD. (B, C) Heat maps showing genes positively and negatively correlated with JAK3 in STAD (Top $50)$. Red indicates positively correlated genes, and green indicates negatively correlated genes. (D-F) Heat map of GO enrichment in CC terms, BP terms, and MF terms. (G) KEGG pathways analysis. GO and KEGG were performed by Gene Set Enrichment Analysis. GO: Gene Ontology; KEGG: Kyoto Encyclopedia of Genes and Genomes; BP: biological process; CC: molecular function; MF: molecular functions. Supplementary Figure 3. KEGG pathway annotations of the cytokine-cytokine receptor interaction. GO and KEGG were performed by Gene Set Enrichment Analysis. Supplementary Figure 4. The enrichment analysis of TYK2 in STAD (LinkedOmics). (A) A Pearson test was used to analyze correlations between TYK2 and genes differentially expressed in STAD. (B, C) Heat maps showing genes positively and negatively correlated with TYK2 in STAD (Top 50). Red indicates positively correlated genes, and green indicates negatively correlated genes. (D-F) Heat map of GO enrichment in CC terms, BP terms, and MF terms. (G) KEGG pathways 
analysis. GO and KEGG were performed by Gene Set Enrichment Analysis. GO: Gene Ontology; KEGG: Kyoto Encyclopedia of Genes and Genomes; BP: biological process; CC: molecular function; MF: molecular functions. Supplementary Figure 5. KEGG pathway annotations of the ribosome. KEGG: Kyoto Encyclopedia of Genes and Genomes. (Supplementary Materials)

\section{References}

[1] M. Venerito, A. C. Ford, T. Rokkas, and P. Malfertheiner, "Prevention and management of gastric cancer," Helicobacter, vol. 25, p. e12740, 2020.

[2] F. Bray, J. Ferlay, I. Soerjomataram, R. L. Siegel, L. A. Torre, and A. Jemal, "Global cancer statistics 2018: GLOBOCAN estimates of incidence and mortality worldwide for 36 cancers in 185 countries," CA: a Cancer Journal for Clinicians, vol. 68, no. 6, pp. 394-424, 2018.

[3] A. Digklia and A. D. Wagner, "Advanced gastric cancer: current treatment landscape and future perspectives," World Journal of Gastroenterology, vol. 22, no. 8, pp. 2403-2414, 2016.

[4] B. Groner and V. von Manstein, "Jak Stat signaling and cancer: opportunities, benefits and side effects of targeted inhibition," Molecular and Cellular Endocrinology, vol. 451, pp. 1-14, 2017.

[5] J. Pencik, H. T. T. Pham, J. Schmoellerl et al., "JAK-STAT signaling in cancer: from cytokines to non-coding genome," Cytokine, vol. 87, pp. 26-36, 2016.

[6] G. Colon, T. Saccon, A. Schneider et al., "The enigmatic role of growth hormone in age-related diseases, cognition, and longevity," Geroscience, vol. 41, no. 6, pp. 759-774, 2019.

[7] S. Gonzalo and N. Coll-Bonfill, "Genomic instability and innate immune responses to self-DNA in progeria," Geroscience, vol. 41, no. 3, pp. 255-266, 2019.

[8] D. E. Johnson, R. A. O'Keefe, and J. R. Grandis, "Targeting the IL-6/JAK/STAT3 signalling axis in cancer," Nature Reviews. Clinical Oncology, vol. 15, no. 4, pp. 234-248, 2018.

[9] M. Forero-Castro, C. Robledo, R. Benito et al., "Mutations in TP53 and JAK2 are independent prognostic biomarkers in Bcell precursor acute lymphoblastic leukaemia," British Journal of Cancer, vol. 117, no. 2, pp. 256-265, 2017.

[10] W. Zhang, H. Zhao, J. Chen et al., "Mining database for the expression and gene regulation network of JAK2 in skin cutaneous melanoma," Life Sciences, vol. 253, p. 117600, 2020.

[11] X. Wang, X. Liao, T. Yu et al., "Analysis of clinical significance and prospective molecular mechanism of main elements of the JAK/STAT pathway in hepatocellular carcinoma," International Journal of Oncology, vol. 55, no. 4, pp. 805-822, 2019.

[12] Z. Tang, C. Li, B. Kang, G. Gao, C. Li, and Z. Zhang, "GEPIA: a web server for cancer and normal gene expression profiling and interactive analyses," Nucleic Acids Research, vol. 45, no. W1, pp. W98-w102, 2017.

[13] D. S. Chandrashekar, B. Bashel, S. A. H. Balasubramanya et al., "UALCAN: a portal for facilitating tumor subgroup gene expression and survival analyses," Neoplasia, vol. 19, no. 8, pp. 649-658, 2017.

[14] A. M. Szász, A. Lánczky, Á. Nagy et al., "Cross-validation of survival associated biomarkers in gastric cancer using transcriptomic data of 1,065 patients," Oncotarget, vol. 7, no. 31, pp. 49322-49333, 2016.
[15] J. Gao, B. A. Aksoy, U. Dogrusoz et al., "Integrative analysis of complex cancer genomics and clinical profiles using the cBioPortal," Science Signaling, vol. 6, no. 269, p. pl1, 2013.

[16] S. V. Vasaikar, P. Straub, J. Wang, and B. Zhang, "LinkedOmics: analyzing multi-omics data within and across 32 cancer types," Nucleic Acids Research, vol. 46, no. D1, pp. D956D963, 2018.

[17] T. Li, J. Fan, B. Wang et al., "TIMER: a web server for comprehensive analysis of tumor-infiltrating immune cells," Cancer Research, vol. 77, no. 21, pp. e108-e110, 2017.

[18] N. O. Siemers, J. L. Holloway, H. Chang et al., "Genome-wide association analysis identifies genetic correlates of immune infiltrates in solid tumors," PLoS One, vol. 12, no. 7, article e0179726, 2017.

[19] P. Danaher, S. Warren, L. Dennis et al., "Gene expression markers of tumor infiltrating leukocytes," Journal for Immunotherapy of Cancer, vol. 5, no. 1, p. 18, 2017.

[20] S. Sousa and J. Maatta, "The role of tumour-associated macrophages in bone metastasis," Journal of Bone Oncology, vol. 5, no. 3, pp. 135-138, 2016.

[21] G. H. Royce, H. M. Brown-Borg, and S. S. Deepa, “The potential role of necroptosis in inflammaging and aging," Geroscience, vol. 41, no. 6, pp. 795-811, 2019.

[22] R. Santín-Márquez, A. Alarcón-Aguilar, N. E. López-Diazguerrero, N. Chondrogianni, and M. Königsberg, "Sulforaphane - role in aging and neurodegeneration," Geroscience, vol. 41, no. 5, pp. 655-670, 2019.

[23] G. Giordano, P. Parcesepe, M. R. D’Andrea et al., "JAK/Stat5mediated subtype-specific lymphocyte antigen 6 complex, locus G6D (LY6G6D) expression drives mismatch repair proficient colorectal cancer," Journal of Experimental \& Clinical Cancer Research, vol. 38, no. 1, p. 28, 2019.

[24] T. A. Waldmann and J. Chen, "Disorders of the JAK/STAT pathway in T cell lymphoma pathogenesis: implications for immunotherapy," Annual Review of Immunology, vol. 35, no. 1, pp. 533-550, 2017.

[25] M. Shen, Z. Xu, W. Xu et al., "Inhibition of ATM reverses EMT and decreases metastatic potential of cisplatin-resistant lung cancer cells through JAK/STAT3/PD-L1 pathway," Journal of Experimental \& Clinical Cancer Research, vol. 38, no. 1, p. 149, 2019.

[26] F. Liang, H. Liang, Z. Li, and P. Huang, "JAK3 is a potential biomarker and associated with immune infiltration in kidney renal clear cell carcinoma," International Immunopharmacology, vol. 86, p. 106706, 2020.

[27] H. Fan, C. Li, Q. Xiang et al., "PIK3CA mutations and their response to neoadjuvant treatment in early breast cancer: a systematic review and meta-analysis," Thoracic Cancer, vol. 9, no. 5, pp. 571-579, 2018.

[28] C. H. Chuang, P. G. Greenside, Z. N. Rogers et al., "Molecular definition of a metastatic lung cancer state reveals a targetable CD109-Janus kinase-Stat axis," Nature Medicine, vol. 23, no. 3 , pp. 291-300, 2017.

[29] M. L. Robinette, M. Cella, J. B. Telliez et al., "Jak3 deficiency blocks innate lymphoid cell development," Mucosal Immunology, vol. 11, no. 1, pp. 50-60, 2018.

[30] E. M. Van Allen, H. G. Golay, Y. Liu et al., "Long-term benefit of PD-L1 blockade in lung cancer associated with JAK3 activation," Cancer Immunology Research, vol. 3, no. 8, pp. 855-863, 2015. 
[31] A. Karjalainen, S. Shoebridge, M. Krunic et al., "TYK2 in tumor immunosurveillance," Cancers, vol. 12, no. 1, p. 150, 2020.

[32] B. Hoesel and J. A. Schmid, "The complexity of NF- $\kappa$ B signaling in inflammation and cancer," Molecular Cancer, vol. 12, no. 1, p. 86, 2013.

[33] W. Seubwai, C. Wongkham, A. Puapairoj et al., "Aberrant expression of NF- $\kappa \mathrm{B}$ in liver fluke associated cholangiocarcinoma: implications for targeted therapy," PLoS One, vol. 9, no. 8, article e106056, 2014.

[34] S. Tan, J. Xu, A. Lai et al., "Curculigoside exerts significant anti-arthritic effects in vivo and in vitro via regulation of the JAK/STAT/NF- $\kappa \mathrm{B}$ signaling pathway," Molecular Medicine Reports, vol. 19, no. 3, pp. 2057-2064, 2019.

[35] J. Świerkot, R. Sokolik, A. Czarny et al., "Activity of JAK/STAT and NF-kB in patients with axial spondyloarthritis," Postępy Higieny $i$ Medycyny Doświadczalnej (Online), vol. 69, pp. 1291-1298, 2015.

[36] A. Kauppinen, T. Suuronen, J. Ojala, K. Kaarniranta, and A. Salminen, "Antagonistic crosstalk between NF- $\kappa \mathrm{B}$ and SIRT1 in the regulation of inflammation and metabolic disorders," Cellular Signalling, vol. 25, no. 10, pp. 1939-1948, 2013.

[37] H. Y. Bako, M. A. Ibrahim, M. S. Isah, and S. Ibrahim, "Inhibition of JAK-STAT and NF- $\kappa$ B signalling systems could be a novel therapeutic target against insulin resistance and type 2 diabetes," Life Sciences, vol. 239, p. 117045, 2019.

[38] L. von Hoff, E. Kärgel, V. Franke et al., “Autocrine LTA signaling drives NF- $\kappa \mathrm{B}$ and JAK-STAT activity and myeloid gene expression in Hodgkin lymphoma," Blood, vol. 133, no. 13, pp. 1489-1494, 2019. 